International Journal of Applied Mathematics

Volume 34 No. $6 \quad 2021,1071-1091$

ISSN: 1311-1728 (printed version); ISSN: 1314-8060 (on-line version)

doi: http://dx.doi.org/10.12732/ijam.v34i6.3

\title{
ON SOME ADVANCES IN DYNAMICS OF ONE VARIABLE COMPLEX FUNCTIONS
}

\author{
Mohammad Sajid \\ Department of Mechanical Engineering \\ College of Engineering \\ Qassim University, Buraidah - 51452 \\ Al Qassim, SAUDI ARABIA
}

\begin{abstract}
In this article, we present some recent advances in the dynamics of one variable complex functions which are especially associated to the Julia sets, the Fatou sets, the Sigel disks, the Herman rings, fixed points as well as the Mandelbrot sets. For the sake of interest, we consider mainly research works which have been done from 2015 to 2019. To achieve our purpose, some interesting and selected themes are considered to show some recent advances in the dynamics of one variable complex functions.
\end{abstract}

AMS Subject Classification: 30D05, 37D45, 37F10, 37F45, 37F50

Key Words: dynamical systems; fixed point; polynomials; rational maps; entire and meromorphic functions; Julia sets; Fatou sets; Sigel disks; Herman rings; Mandelbrot sets; bifurcations; Newton's method

\section{Introduction}

The dynamics of complex functions started in the early 19th century by G. Julia, P. Fatou and S. Lattés but major break through came in this research work in the late 19th century about 1980 after introducing computer graphics into the subject. In the last few decades, the dynamics of complex functions has been studied widely in one dimension and higher dimension by many researchers. Many researchers continue to develop theoretical results and computational techniques for making development in the dynamics of complex functions and

Received: February 6, 2021

(C) 2021 Academic Publications 
it is growing research topic which connects several other fields of scientific research. Details about initial studies and further studies on complex dynamics can be seen in references cited in this paper. History and introductory theory about dynamics of complex functions can be seen in [1, 46, 49, 55, 63].

In this work, we present a review on some recent advances in the dynamics of one variable complex functions as well as highlight some of the most beautiful features. To achieve this goal, we provide the brief description of recent progress on the following themes related to the dynamics of one variable complex functions which has been done during 5 years from 2015 to 2019: Simply connected invariant Fatou component, the Julia set's connectivity of Newton's method, dynamical properties about Halley map or Schroder map, poly-time computable Julia set, dynamical properties of the family of degree 4 Blaschke products, Siegel disks of polynomials, quasisymmetric geometry, shapes of polynomial Julia sets, buried Julia components, parabolic-like mapping and its dynamical properties, McMullen-like mapping as a rational map, unmating of rational maps, the bungee set of a quasiregular map, the structure MandelbrotSierpinski maze, the Hausdorff measure's formula on the Julia set using singular traces, biaccessibility dimension, quasi-periodic trajectory.

\section{Different approaches to studying dynamics of one variable complex functions}

Our focus is to explore some interesting recent features of the dynamical behaviour of functions in one-dimensional complex plane. The following different approaches to studying dynamics of one variable complex functions have been found in the literature:

We begin with results on invariant Fatou components. For a meromorphic function $f: \mathbb{C} \rightarrow \hat{\mathbb{C}}$, the boundary behaviour of map $f$ is deeply described in [5] on its invariant simply connected Fatou component. There is a case especially considered when the map is non-rational or transcendental. The theory of accesses to boundary points of $U$ as well as its relation to the dynamics of $f$ develops as a prime goal. There is purposely established a consistency among invariant accesses from $U$ to $\infty$ or weakly repelling points of map $f$ as well as boundary fixed points of the related inner function on $\mathbb{D}$. A dynamical form of the Correspondence Theorem is also shown which explains the relation among the topology of accesses and boundary behaviour of the Riemann map. Moreover, it realizes the structure of $J(f)$ near $\infty$ of a Newton map. As an application of above results, for the Newton maps, a description about the 
accesses to $\infty$ is provided from invariant Fatou components.

When Newton's method uses to a family of maps that has multiple root, a jump in degree and complexity arise as the parameters vary. For Newton's method, a unified approach about connectivity of the Julia sets of holomorphic function discuss by Baranski, Fagella, Jarque and Karpinska [6]. This work is mainly divided in three parts. In the part one, the historical introduction about Newton's method and connectivity of the Julia set is given. In the 2nd part, terminology using in the proofs and preliminary results are mentioned. The proof of the major outcome is shown in the third part. The result on the simple connectivity is provided for case of finite and infinite degree. An approach using in the proof is based on [4] which is introduced by authors. Assuming relative positions of certain sets, the existence of fixed points of the map as well as, under iteration of the map, their images are provided. The existence of absorbing domains inside Baker domains establishes here. It is noted that use these new ideas for all range of Fatou components which is not restricted to Baker domains only. In the third part, $N$ denotes a Newton map which arises by applying the Newtons method to a transcendental entire function or a polynomial. The proof of the main result is shown very rigorously as every Fatou component $U$ of $N$ is simply connected. Apart from above, these results also establish:

(i) $N$ has no invariant Herman rings.

(ii) An invariant Baker domain $U$ of $N$ possess a simply connected absorbing domain.

(iii) Suppose $U$ is either a periodic Fatou component of minimal period greater than one, a preperiodic component or a wandering domain, then $U$ is simply connected.

Moreover, $U$ cannot be a $p$-periodic Herman ring. These proofs are applicable in all similar situations.

Can we choose any set of $n$ distinct points in the complex plane and construct a polynomial in order to those $n$ points form an attracting cycle for the related Halley map $H_{p}(z)$ or Schroder map $S_{p}(z)$, where

$H_{p}(z)=z-\frac{2 p(z) p^{\prime}(z)}{2 p^{\prime}(z) p^{\prime}(z)-p(z) p^{\prime \prime}(z)}$ and $S_{p}(z)=z-\frac{p(z)}{p^{\prime}(z)}-\frac{p(z) p(z) p^{\prime \prime}(z)}{2 p^{\prime}(z) p^{\prime}(z) p^{\prime}(z)} ?$

If yes, what is the minimal degree of this type of polynomial? The work by Collins [20] is mainly focus on these questions: To achieve this goal, for each 
$n \geq 2$, it proves that for any set of $n$ distinct points in $\mathbb{C}, \exists$ a polynomial $p$ of degree $\leq n+1$ then the corresponding $H_{p}(z)$ and $S_{p}(z)$ have the given points as a super-attracting cycle. It improves the result given in [3], which presents how to determine that type of a polynomial of degree $3 n$. In addition, it demonstrates that this degree estimate changes from $3 n$ to $n+1$. By examples, it is constructed rigorously to explain that each of the above degree estimate is sharp for both Halley map $H_{p}(z)$ and Schroder map $S_{p}(z)$.

A poly-time computable Julia set having a recurrent critical point discuss in [24] and it is claimed that it is first example and no examples are known in literature till this work. To represent this work, the very well known quadratic map is considered which is the Feigenbaum polynomial $f_{c_{*}}$. It is shown that the Julia set of the Feigenbaum map is computable in polynomial time. To resolve the problem, the poly-time computability of the Julia set of the Feigenbaum renormalization fixed point $F$ is proved. This map $\mathrm{F}$ is quadratic-like and conjugate to $f_{c_{*}}$, as well as the Julia set of $F$ is homeomorphic to that of $f_{c_{*}}$. The introduction of the concept of an oracle is given to define computability of real or complex functions.

For the Blaschke products $B_{a}(z)=z^{3} \frac{z-a}{1-\bar{a} z}$ of degree 4, the dynamical properties are investigated in [15]. The concept of tongues for $B_{a}$ is introduced and focus on the tongue-like sets. Proofs of the non-emptiness of tongue, connectivity of tongues and the boundary of a tongues are provided. The tongues are bounded for $|a|<3$. The Blaschke product $B_{a}$ has a parabolic cycle of multiplier 1 if $a$ lies to the boundary of a tongue. For the parameter plane, there are visible some symmetries. Further, the description of the bifurcation is given which take place in a neighborhood of the tips of the tongues. It observes that if the parameter is very near to a tip, the two cycles leaving the unit circle are attracting. The Blaschke product $B_{a}$ possess a parabolic cycle on the unit circle and if $|a|>2$, then $a$ lies to the boundary of a tongue. The shape of the connected components of the extended fixed tongue is also described. It is seen that the extended fixed tongue is symmetric with respect to the real line. Moreover, by observing numerical computation, the properties obtained are common to all extended tongues for the extended fixed tongue. The following two conjectures on all the extended tongues are mentioned:

Conjecture 1: Given an extended tongue $E T_{\tau}$ of period $p>1$, its connected components are disjoint. The boundary of every connected component of the extended tongue $E T_{\tau}$ consists of two disjoint connected components. The exterior component consists of parameters for which there is a parabolic cycle of period $p$ and multiplier 1 . The interior component consists of parameters for which there is a parabolic cycle of period $p$ and multiplier -1 . Moreover, there 
is a period doubling bifurcation taking place throughout the curve of interior boundary parameters.

Conjecture 2: Parabolic curves accumulate on the unit circle on isolate points and tangentially to it. Moreover, if a parabolic curve accumulates on a parameter $l,|l|=1$, then there is another parabolic curve of the same period landing on $l$ from the opposite side.

These conjectures have a similar structure as the one given in the following proposition as: Let $E T_{0}$ denote the extended fixed tongue which intersects the real line. Then, $E T_{0}$ satisfies the following properties: (a) $E T_{0}$ is symmetric with respect to the real line. (b) For fixed $r_{0}, 1<r_{0}<2, E T_{0} \cap\{\alpha \geq 0\} \cap\{r=$ $\left.r_{0}\right\}$ is a connected set on which the multiplier is strictly increasing with respect to $\alpha$ and takes values in $(b, 1)$, where $-1 \leq b<0$. Moreover, $b=-1$ if and only if $r_{0} \leq 5 / 3$. (c) If $(r, \alpha) \in E T_{0}$, then $-1 / 6<\alpha<1 / 6$.

Apart from the above, for Blaschke products $B_{a, \lambda}(z)=z^{3} \frac{z-a}{1-\bar{a} z}+\frac{\lambda}{z^{2}}$, the singular perturbations and connectivity of Fatou components are studied by Canela [14]. Under certain conditions, it is found that all Fatou components of $B_{a, \lambda}(z)$ have finite connectivity. Further, $J\left(B_{a, \lambda}\right)$ is the union of countably many Cantor sets of quasicircles as well as uncountably many point components.

A theorem of Herman extends in [18] as Siegel disks of polynomials with 2 finite critical values contain essentially a critical point on their boundaries under a diophantine condition on the rotation number. It is known for unicritical polynomials. Suppose $\mathcal{H}$ is the set of $\theta \in \mathbb{R}$ such that, for rotation number $\theta$, every orientation-preserving analytic circle diffeomorphism is analytically conjugate to a rotation. It is shown that there is an element in the cycle of Siegel disk $\Delta$ whose boundary holds a critical point for every polynomial with 2 finite critical values, and $\Delta$ of arbitrary period and of $\theta$ in $\mathcal{H}$. A proof of the conjecture on rational maps is provided as: The boundaries of $\Delta$ of rational maps hold a critical point whenever $\theta$ belongs to $\mathcal{H}$. It is mentioned that this theorem extended by Benini and Fagella [8] for the case of transcendental entire maps.

In [64], a result on Siegel disk is rigorously shown as: For $0<\theta<1$, the boundary of the Siegel disk of $f_{\theta}(z)=e^{2 \pi i \theta} \sin (z)$ centered at the origin is a Jordan curve which goes through exactly 2 critical points $\pi / 2$ and $-\pi / 2$. Here $\theta$ refers as of Petersen-Zakari (PZ) type and $\left[a_{1}, a_{2}, \ldots, a_{n}, \ldots\right]$ is its continued fraction. If $\sup \left\{a_{n}\right\}<\infty$, then $\theta$ is of bounded type . To prove this result, David homeomorphisms as well as David's integrability theorem are introduced. For some Blaschke models, there is proposed a route to justify the integrability of the invariant Beltrami differentials for which Petersen's puzzle construction is unavailable. The construction of an odd Blaschke fraction is provided which 
perform as the model map for $f_{\theta}$. The proof of the above result contains three key parts as variant of Vitali's covering lemma, concept of K-admissible pair and group of dynamically defined domains. Yoccoz's cell construction is given and uses its geometry.

For the Julia set of rational maps, the quasisymmetric geometry of Cantor circles describes theoretically and analytically, as well as by pictures using particular numerical values in [53]. This work explores many interesting mathematical computation and calculation to compute the Cantor circles as the Julia set. Three families of parabolic rational maps consider here. It is shown that, for suitable parameters, every Cantor set of circles as the Julia set of a nonhyperbolic rational map must be quasisymmetric equivalently the Julia set of one map from above 3 families. A complete classification of quasisymmetric of the Cantor circles as the Julia sets of rational maps is provided. The construction of the families of non-hyperbolic rational maps are given for which Julia sets are Cantor circles with 1 parabolic fixed point, 2 parabolic fixed points and parabolic periodic points. A sufficient and necessary condition is established whenever a component of a Cantor circles Julia set is a quasicircle. Further, for McMullen maps $f_{\lambda}(z)=z^{m}+\frac{\lambda}{z^{l}}, \lambda \in \mathbb{C} \backslash\{0\}$, the quasisymmetric geometry of the Julia sets of $f_{\lambda}(z)$ is discussed in [54], where $l$ and $m$ are positive integers satisfying $\frac{1}{l}+\frac{1}{m}<1$.

The research work [39] is based on the theme that fix the shape which needs to approximate, thereafter change the degree of the polynomials for which Julia sets approximate the shape. Using the Hausdorff metric, it is represented that, in the complex plane, any Jordon curve approximates arbitrarily in the Hausdorff topology by Julia sets of polynomials. By combining these polynomials, there is constructed rational maps whereof basins of attraction estimate much complicated sets. Interestingly, the generated shapes of filled Julia sets look like cat, butterfly and heart.

In [40], the parabolic-like mapping is defined as well as studies its dynamical properties. This type of map is a local concept, which is depicted by a filled Julia set as well as by an external map. Further, a parabolic-like map possess a parabolic fixed point with an attracting petal outside the filled Julia set, while the external map of a parabolic-like mapping contains a parabolic fixed point. The theory of polynomial-like mappings is mainly expanded to parabolic-like mappings. Rigorously, the Straightening Theorem is proved as any paraboliclike mapping of degree 2 is hybrid conjugate to a member of the family

$$
\operatorname{Per}_{1}(1)=\left\{\left[P_{A}\right] \mid P_{A}(z)=z+\frac{1}{z}+A, A \in \mathbb{C}\right\}
$$

a unique such member if the filled Julia set is connected. It is given that all of 
the maps in $\operatorname{Per}_{1}(1)$ have a completely invariant Fatou component.

In [29], the prime goal of work answers the question that is raised by McMullen [42] who provides rational maps family of degree 3 with explicit formula that is not belonging to the family of rational maps $g_{c, \lambda}: z \mapsto z^{d_{\infty}}+c+\frac{\lambda}{z^{d_{0}}}$, where $d_{\infty}, d_{0} \geq 1$ and $c, \lambda \in \mathbb{C}$. Moreover, those Julia set exhibits buried Julia components of various types: points, Jordan curves; however, also Julia components which are neither points nor Jordan curves. It is important to be studied that the buried Julia components does not belong to the disconnected Julia set of any polynomial map. For rational maps, the buried Julia components may arise. Specially, here focuses on disconnected case. The main target is to introduce a family of rational maps arising from a special tree that is to answer the question of McMullen. The dynamical properties are stated in the following result: If the following conditions satisfy by the weighted dynamical tree $\left(\mathcal{H}_{P}, w\right)$ :

$$
\widehat{d}=\frac{1}{2}\left(d_{0}+d_{1}+d_{2}-1\right) \text { is an integer } \geq 2 \text { and } \max \left\{d_{0}, d_{1}, d_{2}\right\} \leq \widehat{d}
$$

$$
\left(\mathcal{H}_{P}, w\right) \text { is unobstructed, }
$$

then, $\exists$ a rational map $f$ of degree $\widehat{d}+d_{3}$ such that the exchanging dynamics of postcritically separating Julia components of $f$ is encoded by $\left(\mathcal{H}_{P}, w\right)$ in the following sense: (i) every critical orbit accumulates a super-attracting cycle $\left\{z_{0}, z_{1}, z_{2}, z_{3}\right\}$ of period 4; (ii) $\exists$ a homeomorphism $h: \mathcal{J}_{\text {crit }}(f) \rightarrow \mathcal{J}\left(\mathcal{H}_{P}\right)$ such that the following diagram commutes

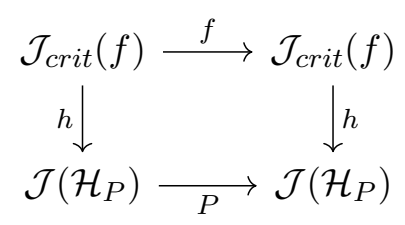

(iii) for every Julia component $J \in \mathcal{J}_{\text {crit }}(f)$ such that $h(J)$ is not eventually mapped under iteration to the fixed branching point $\alpha,\left.f\right|_{J}$ has degree $w\left(e_{k}\right)=$ $d_{k}$ where $e_{k}$ is the edge of $\mathcal{H}_{P}$ which contains $h(J)$.

Further, the result related to the topological properties of the Julia component of function is given as: For assuming (A1) and (A2), $\exists$ a rational map $f$ which satisfies the above result as well as $J(f)$ contains buried Julia components of many types: (a) Point type: uncountably many points. (b) Circle type: uncountably many Jordan curves. (c) Complex type: countably many preimages of a fixed Julia component lying over the fixed branching point $\alpha$, 
say $J_{\alpha}=h^{-1}(\alpha) \in \mathcal{J}(f)$, which is quasiconformally homeomorphic to the connected Julia set of a rational map $\hat{f}$.

Furthermore, $\widehat{f}$ has degree $\widehat{d}$ and has only one critical orbit which is a superattracting cycle $\left\{\widehat{z_{0}}, \widehat{z_{1}}, \widehat{z_{2}}\right\}$ of period three such that the local degree of $\widehat{f}$ at $\widehat{z_{k}}$ is $d_{k}$ for every $k \in\{0,1,2\}$.

A unifying example by giving a McMullen-like mapping as a rational map related to a hyperbolic postcritically finite polynomial $P$ and a pole data $D$ mentions in [27] that encodes the location of every pole of the rational map and the local degree at each pole. All these examples present parameter values, where a Cantor set of circles is exhibited in the associated Julia set. To achieve this goal, a generalization of special dynamical behavior introduces for rational map $f$. The characterization of a McMullen-like mapping by applying an arithmetic condition depending only on $(P, D)$ is given as for the McMullen family $f$. It is provided that the arithmetic condition is necessary. For a McMullen-like mapping of minimal degree four, an explicit expression is given. The following two examples obtain topologically conjugate on their Julia set to the McMullen family for McMullen-like mappings.

Example 1: Suppose the family of rational maps given by $\widetilde{f}_{\lambda}(z)=z^{n}+$ $\lambda /(z-a)^{d}$ and fix a small value of $\lambda \neq 0$. For every $|a|$ sufficiently small, if the arithmetic condition $1 / n+1 / d<1$ is satisfied, then $\widetilde{f}$ is a McMullen-like mapping of same type as $f_{\lambda}$. In this case the pole is moved to $a$ and not located at the origin as in the McMullen family. The Julia set $\mathcal{J}\left(\widetilde{f}_{\lambda}\right)$ is a Cantor set of circles.

Example 2: Consider a family of rational maps given by $\widehat{f}_{\lambda}(z)=z^{n}+$ $\Lambda(z) / z^{d}$ where $\Lambda(z)$ is any polynomial with $\operatorname{deg}(\Lambda)<d$ and $\Lambda(0) \neq 0$ small enough, if the arithmetic condition $1 / n+1 / d<1$ is satisfied, then $\widehat{f}$ is a McMullen-like mapping of same type as $f_{\lambda}$.

The mating and unmating of rational maps provide in [45]. This work contains two-way (dual) attempts on unmating of rational maps which is based on the operation of mating of two polynomials by Douady and Hubbard [23]. A sufficient condition is given that the rational map come up as a mating of two polynomials. The two opposite cases of rational maps study here and is contrasted hyperbolic maps where the Fatou set holds all critical points, with maps where the Julia set holds all critical points. A stronger version of Moore's theorem is provided, and then the mating construction is given. The sufficient criterion presents in $[43,44]$ for rational map to arise as a mating for rational maps. The algorithm that unmates rational maps is also given. Many examples describe for unmating of rational maps. It is shown by using example that the existence of a pseudo-equator is not essential for a rational map to arise 
as mating. The following open questions related to mating of rational maps propose in this work:

- Suppose $f$ is a rational map from $\widehat{\mathbb{C}}$ to $\widehat{\mathbb{C}}$. Is the existence of an equator for $f$ sufficient for $f$ to arise as a mating? When $f$ is postcritically finite, which is more expected, but there does not seem to be a proof in the literature.

- A (pseudo-) equator $\mathcal{E}$ has to be orientation-preserving (pseudo-) isotopic to $f^{-1}(\mathcal{E})$. Orientation-reversing (pseudo-) equators seem to be as common as orientation- preserving ones. From such a pseudo-equator it is possible to construct a semi-conjugacy from $z^{-d}: S^{1} \rightarrow S^{1}$ to the map $f$, where $d=\operatorname{deg} f$ (when $f$ is postcritically finite and $\mathcal{J}(f)=\widehat{\mathbb{C}}$ ). There should be some sort of orientation-reversing mating associated to such orientation-reversing (pseudo-) equators.

- The form of Moore's theorem presented here allows one to shrink each ray-equivalence class to a point by a pseudo-isotopy $H$. Is it possible to put further smoothness assumptions on $H$ ? In particular, can one embed $H$ in a holomorphic motion?

Further, a continuity consequence on quadratic matings discusses in [41] with respect to parameters of odd denominator rationals. The mating the basilica with a Siegel disk is found in [62] by considering a family of rationals maps $R_{a}(z)=\frac{a}{z^{2}+2 z}$, where $a \neq 0$ is a complex parameter. Moreover, in [59], the matings of cubic polynomials with a fixed critical point is explored.

For a quasiregular map of transcendental type $f: \mathbb{R}^{n} \rightarrow \mathbb{R}^{n}$, the bungee set studies first time in [50] by Nicks and Sixsmith. The bungee set is given as the set points for which orbit is neither bounded nor tends to $\infty$. It is defined as

$$
B U(f):=\mathbb{C} \backslash(I(f) \cup B O(f)),
$$

where $I(f)$ is the escaping set and $B O(f)$ is the bounded orbit set. It is proved that the bungee set is infinite, as well as it shares several properties from $B U(f)$ of a transcendental entire function. It is shown that $B U(f) \cap J(f)$ is an infinite set further, as a conjecture, there mentions that $J(f)$ possesses positive capacity under certain conditions. For $f: \mathbb{R}^{2} \rightarrow \mathbb{R}^{2}$, it is found that $\operatorname{cap} J(f)$ is strictly positive as well as $J(f) \neq \partial B U(f)$. In the quasiregular setting, the examples of some new properties of the bungee set are provided. For instance, a quasiconformal map of the plane is given with a non-empty $B U(f)$; but for an analytic homeomorphism, this behaviour is not possible. 
The structure MandelbrotSierpinski maze (MS-maze) explains by Devanay in [22] for family of rational maps $z^{2}+\lambda / z^{3}$. It is a newest structure that belongs to family of these functions in the parameter plane. In the first phase of the construction, the beginning portion of the MS-maze is constructed which is called Sierpindelbrot arc (SM arc). At this first level, the maze consists an infinite string of alternating Mandelbrot sets as well as Sierpinski holes that consist along an arc in the parameter plane. In this construction, the concept of a polynomial-like map uses. The Mandelbrot sets $\left(\mathcal{M}^{k}\right)$ and Sierpinski holes $\left(\mathcal{E}^{k}\right)$ arrange as:

$$
\ldots \mathcal{M}^{4}<\mathcal{E}^{3}<\mathcal{M}^{3}<\mathcal{E}^{2}<\mathcal{M}^{2}<\mathcal{E}^{1} .
$$

where $k(\geq 2)$ is the period of the attracting cycle for parameters take out from the main cardioid of $\mathcal{M}^{k}$ as well as $k(\geq 1)$ is the escape time in $\mathcal{E}^{k}$.

In the second phase, infinitely many smaller $\mathcal{M}^{k}$ and $\mathcal{E}^{k}$ that alternate on the arc between each $\mathcal{M}$ and $\mathcal{E}$ on the first level, as well as finitely many other $\mathcal{M}^{k}$ and $\mathcal{E}^{k}$ that extend away from the given Mandelbrot set in a pair of different directions.

In the final phase, this completes the construction of MS-maze. In this final level, repeat inductively the above structure to generate the Mandelpinski maze. Further, this work extends from the construction of the Mandelpinski maze to a family $z^{n}+\lambda / z^{d}$, where $n(\geq 2)$ is even but $d(\geq 3)$ is odd.

In [21], a complete rigorous proof of a formula proves for the Hausdorff measure on the Julia set in terms of singular traces. Moreover, there is reconstructed proofs of some existing results for resolving their purposes. It is proved that if $\mathcal{C}$ is any Jordan curve in $\mathbb{C}$ with finite upper $s$-dim Minkowski content, and $\zeta$ is a conformal equivalence between the exterior of $\mathbb{D}$ and the exterior of $\mathcal{C}$, then $\left[F, M_{\zeta}\right]$ is in the weak Schatten ideal $\mathcal{L}_{s, \infty}$. Further, it is shown that $f \mapsto \varphi\left(M_{f \circ Z}\left|\left[F, M_{Z}\right]\right|^{p}\right)$ is $p$ - conformal with respect to $\phi_{c}$. Furthermore, the self-contained proof of the following result is given: Suppose $f$ is a complex polynomial. Then,

(1) $\left[F, f\left(M_{Z}\right)\right]-f^{\prime}\left(M_{Z}\right)\left[F, M_{Z}\right] \in\left(\mathcal{L}_{p, \infty}\right)_{0}$;

(2) $\left|\left[F, f\left(M_{Z}\right)\right]\right|^{p}-\left|f^{\prime}\left(M_{Z}\right)\right|^{p}\left|\left[F, M_{Z}\right]\right|^{p} \in\left(\mathcal{L}_{1, \infty}\right)_{0}$.

Moreover, some familiar results on the connection between Dixmier traces and zeta-function residues are also provided. Using these results, the following result gets: Suppose $c \neq 0$ is in the main cardioid of the Mandelbrot set and $p \in(1,2)$ is the Hausdorff dimension of the Julia set $J$ of $\phi_{c}$. "Suppose $m_{p}$ is the $p$-dim Hausdorff measure on $J$. Then, 
(i) $\left[F, M_{Z}\right] \in \mathcal{L}_{p, \infty}$;

(ii) $\forall$ continuous Hermitian trace $\varphi$ on $\mathcal{L}_{1, \infty}, \exists$ a constant $K(\varphi, c)$ such that for every $f \in C(J)$,

$$
\varphi\left(M_{f \circ Z}\left|\left[F, M_{Z}\right]\right|^{p}\right)=K(\varphi, c) \int_{J} f d m_{p} ;
$$

(iii) if $\omega$ is a dilation-invariant extended limit on $L_{\infty}(0, \infty)$ such that $\omega \circ \log$ is also dilation invariant, then $K\left(t r_{\omega}, \phi_{c}\right)>0$. Here, $t r_{\omega}$ is a Dixmier trace corresponding to $\omega . "$

The biaccessibility dimension is given as the Hausdorff dimension of those external angles whose the corresponding rays land together with some other ray. In [13], a discussion about a purely combinatorial characterization of biaccessible angles provides for both dynamic and parameter forms, and the Hausdorff dimension of the set of biaccessible angles estimate here. Only the case of quadratic polynomials $p_{c}=z^{2}+c$ is considered in this work. The following two sets investigate

(i) $\mathcal{B i a c}_{\vartheta}$ the set of combinatorially biaccessible $\varphi \in \mathbb{S}^{1}$ with respect to $\vartheta$

(ii) $\mathcal{B}$ iac the set of combinatorially biaccessible parameter angles $\vartheta \in \mathbb{S}^{1}$, where $\vartheta$ is the parameter angle.

Based on these sets, the Hausdorff dimension of biaccessible angles $\operatorname{dim}_{H}\left(\mathcal{B i a c}_{\vartheta}\right)$ and the Hausdorff dimension of biaccessible parameter angles $\operatorname{dim}_{H}(\mathcal{B} i a c)$ are discussed. It is proved that for every parameter angle $\vartheta \in \mathbb{S}^{1}$, $\operatorname{dim}_{H}\left(\mathcal{B i a c}_{\vartheta}\right)$ for $N=N(\nu(\vartheta))$ and $S=S_{\kappa+1}(\nu(\vartheta))$.

Further, the dimension of renormalizable angles and kenading sequences are described. An algorithm from internal address to kneading sequence is given. Topologically and combinatorially biaccessible angles in the Julia set and the Mandelbrot are expressed. The two pairs of bounds are given for the Hausdorff dimension of biaccessible itinearies and kneading sequences. Furthermore, the embedding of the Hubbard tree utilize in their computations.

In [38], the synchronous chaos discusses on the Julia set of complex-valued coupled map lattices. It is very interesting application of chaotic set which is called the Julia set in the complex plane. Due to complex-valued coupled map lattices, it has become an important study since the local synchronization is found by some researchers for real coupled map lattices. Firstly, a unified formulation is given for describing both local and global synchronization theory of complex-valued coupled map lattices. Secondly, investigates the problem of 
constructing a network for which the fastest synchronized speed can be made by choosing suitable coupling coefficients.

Further, solve an inf min max problem related to the problem of finding a complex coupling weight among a class of coupling matrices which gives the fastest synchronized rate. Moreover, the global and local synchronization are shown on the Julia set with individual maps of the quadratic and cubic forms. The important and interesting statistical properties of real and complex onedimensional maps are discussed in [56] under weak hyperbolicity assumptions which are very useful in the study of Julia sets, fractal dimensions, etc. The existence and statistical properties of physical measures is studied, with respect to geometric reference measures. The geometric properties of these measures are also found. The reference measure $\mu$ could be the Lebesgue measure on the phase space, or more generally a conformal measure supported on the Julia set. Such a measure $\nu$, when ergodic, has the important property of being a physical measure with respect to $\mu$. The dynamics of $J(f)$ is taken, where the chaotic dynamical behavior concentrates.

For smooth interval maps, Bruin, Luzzatto and van Strien [12] give mixing rates upper bounds closely related to the growth of derivatives at the critical values. But the results from this work express that the mixing rates can be much faster than the growth of derivatives at critical values: an interval map $f$ satisfying the Large Derivatives condition

$$
\lim _{n \rightarrow \infty}\left|D f^{n}(v)\right|=\infty \text {, for each critical value } v \text { of } f \text { in the Julia set }
$$

together with other mild conditions, has a super-polynomially mixing absolutely continuous invariant measure. A result shows in the complex setting for a non-renormalizable polynomial. For a general rational map without parabolic cycles, it is proved that the summability condition with exponent 1 is enough to guarantee the existence of a super-polynomially mixing absolutely continuous invariant measure. The two results are proved, one case for real as well as another for complex. It is also shown that a map satisfying the some assumptions, has the following two expanding properties: expansion away from critical points as well as backward contraction. Verbally, the first property means that outside any given neighborhood of the critical points the map is uniformly hyperbolic; the second property means that a return domain to a ball of radius $\delta$ centered at a critical value is much smaller than $\delta$. A finer quantification of the expansion features of a map is given that satisfies these two properties.

It is shown in [30] that the components of the preimages of a small ball intersecting the Julia set shrink at least at a super-polynomial rate. This unexpected result represents a significant improvement on the estimate of the same 
type for rational maps which satisfies the summability condition with a sufficiently small exponent. It is deduced that the Julia set is locally connected when it is connected. Next, a dimension-like parameter that measures the combined size of all "bad pull-backs" of a suitably chosen small neighborhood of the critical points is introduced. A bad pull-back is a pull-back that is not contained in any diffeomorphic pull-back. Using the local Markov structure (nice sets) provided by the backward contracting property, it is shown that the badness exponent is zero. A straight outcome of this result is that the conical Julia set has codimension zero in the Julia set. A tail estimate in terms of the rate of shrinking of components of preimages of small sets is obtained, and of the badness exponent only. The result on the regularity of the invariant density is found through an upper bound of the Poincare series. For a complex polynomial that is expanding away from critical points and backward contracting, it is shown that the Julia set has Hausdorff dimension $<2$ and is holomorphically removable.

In [51], new results related to Eremenkos conjecture and Bakers conjecture, and about the rate of escape in Baker domains are obtained. In progress on Eremenkos conjecture, that all the components of $I(f)$ are unbounded, and on Bakers conjecture, that if $f$ has order at most $1 / 2$, minimal type, then all the components of $F(f)$ are bounded. Some examples are provided by considering the functions (i) $f(z)=2 z\left(1+e^{-z}\right.$ ) (ii) $f(z)=z+1+e^{-z}$ (iii) $f(z)=z+b \sin z$, where $b>2 \pi$ (iv) $g(z)=2 z \cos \sqrt{z}$.

The concepts of certain polynomial perturbations and uniform linearizability introduce in [28]. There is also mentioned the concept of $J$-stability for polynomial-like maps. It has a direct result relating $J$-stability, irrationally indifferent periodic points, and uniform linearizability. It is known that every irrationally indifferent fixed point of an analytic function with a Brjuno rotation number is linearizable, and this is sharp for quadratic polynomials. The Brjuno numbers set is given by

$$
\mathcal{B}=\left\{\alpha \in \mathbb{R} \backslash \mathbb{Q}: \Sigma q_{n}^{-1} \log q_{n+1}<\infty\right\} .
$$

In the complex plane, an analytic function $f(z)=\lambda z+O\left(z^{2}\right)$ defined in a neighborhood of zero is linearizable near the fixed point 0 if it is locally analytically conjugate to its linear part. This work attentions toward the irrationally indifferent case where $\lambda=e^{2 \pi i \alpha}$ with $\alpha$ irrational. Douady conjectured as this is sharp for all rational functions of degree $\geq 2$. It is proved that Douadys conjecture holds for the class of polynomials for which the number of infinite tails of critical orbits in the Julia set equals the number of irrationally indifferent cycles. The following result is also given as: If family $f(a, z)=\lambda z+\sum_{k=2}^{\infty} f_{k}(a) z^{k}$ is 
uniformly linearizable for $|a| \leq r$, then the quadratic polynomial $F(z)=\lambda z+z^{2}$ is linearizable. There also provides a result on the persistence of Siegel disks and Cremer points on the $J$-stable set. Saturated polynomials are those for which the Fatou-Shishikura inequality on the number of non-repelling cycles is an equality. It is established that saturated polynomials do not have exotic Siegel disks. Moreover, it is found that Julia-saturated polynomials do not have exotic Siegel disks.

For the family of polynomials $P_{c}(z)=z^{n}+c z^{k}, n>k \geq 2$, where $c$ is a complex parameter, the geometric limits of Julia sets and connectedness locus of $P_{c}(z)$ study in [2] with $n$ and $k$ positive integers. The connectivity of the Julia sets of singularly perturbed rational maps $f_{\lambda}(z)=\frac{z^{n}\left(z^{2 n}-\lambda^{n+1}\right)}{z^{2 n}-\lambda^{3 n-1}}, n \geq 2$, $\lambda \in \mathbb{C}^{*}-\left\{\lambda: \lambda^{2 n-2}=1\right\}$; explores in [25]. Bergweiler [11] provides criteria for the escaping set and the Julia set of an entire function to have positive Lebesgue measure. For transcendental meromorphic functions, the dynamics of a family shows in [58] and Herman rings with small periods as well omitted values determine in [16]. The dynamical properties of the extraneous fixed points characterize in [26] with respect to their stability, basins of attraction, cycles, etc. Moreover, the graphical visualizations of the corresponding Fatou and Julia sets also demonstrate. In [33], the dynamics and limiting behavior of Julia sets of König's method for multiple roots are given by showing, for all order $\sigma \geq 2, \exists$ a complex polynomial such that the Julia set of König's method for multiple roots applied to it, is disconnected. In [19], there is relationship given as: (i) The Julia set of orthogonal polynomials to the outer boundary of the support. (ii) The filled Julia set to the polynomial convex hull of the support. (iii) The Green function associated with orthogonal polynomials to the Greens function for the complement of the polynomial convex hull.

The topological properties of Fatou sets and Julia sets of transcendental entire functions little widely discuss in [37] which is translated research work from [36]. The dynamics of two parameter families of hyperbolic sine and cosine like functions investigates in $[9,10]$. It is shown in [60] that Fatou sets, Julia sets, and escaping sets of a transcendental semigroup are respectively equal to the Fatou sets, Julia sets, and escaping sets of their subsemigroups under some conditions. An explicit construction of polynomial semigroups of two generators with a Cantor target as a Julia set is given by Morosawa [48]. Further, it is found that the existence of polynomial semigroups such that the associated Julia set contains no interior points as well as Hausdorff dimension arbitrarily close to 2. A criterion studies in [61] for some rational maps having exactly one fixed (super-) attracting or parabolic basin that provides guarantee the existence of the carpet Julia sets. 
The exploration of filled-in Julia sets arising from centered polygonal lacunary functions find in [47]. It is determined in [7] that Newton maps of rational functions are conjugate to quadratic polynomials. Further, it is provided that there are no Newton maps of rational functions which are conjugate to $z^{3}$. The dynamics and Julia sets of iterated elliptic functions present in [31] by considering the parametrized family of elliptic functions $F_{\Lambda, b}(z)=\wp(z)+b$ for $b \in \mathbb{C}$, $\Lambda$ a lattice, and $\wp_{\Lambda}$ the Weierstrass elliptic $\wp$ function with period lattice $\Lambda$. In [32], Hittmeyer, Krauskopf and Osinga explore interactions of the Julia set with critical and (un)stable sets in an angle-doubling map on $\mathbb{C} \backslash\{0\}$ by studying a nonanalytic perturbation of the complex quadratic family $z \mapsto z^{2}+c$ in the form of a two-dimensional noninvertible map. In [34, 35], it is obtained that there exists a transcendental entire function whose Julia set contains quasiconformal copies of the given quadratic Julia sets for any finite as well as infinite collection of quadratic Julia sets respectively. In [52], the radial distribution of Julia sets of some entire functions with infinite lower order is studied which are solutions, the polynomial or differential polynomial of solutions of the equation $f^{\prime \prime}(z)+A(z) f^{\prime}(z)+B(z) f(z)=0$. Some dynamical properties of fundamental trigonometric functions $\sin z, \cos z$ and $\tan z$ have been found in [57]. Fractals from nonlinear iterated function system of the complex mapping family $f(z)=z^{n}+c$ are generated in [17].

\section{Conclusion}

In the present work, we have been presented some recent advances in the dynamics of one variable complex functions as well as explored beautiful features from 2015 to 2019. It is seen that there are several different approaches in dynamics of one variable complex functions but there are still many more possibilities of research work in this field. Here we have been restricted our self to some selected research themes in some certain directions.

\section{References}

[1] D.S. Alexander, F. Iavernaro, and A. Rosa, Early Days in Complex Dynamics: A History of Complex Dynamics in One Variable During 1906-1942, History of Mathematics, Volume 38, American Mathematical Society and London Mathematical Society (2011).

[2] A.M. Alves, Geometric limits of Julia sets and connectedness locus of the 
family of polynomials $P_{c}(z)=z^{n}+c z^{k}$, Dyn. Syst., 34, No 4 (2019), 668684; https://doi.org/10.1080/14689367.2019.1593321.

[3] S. Amat, S. Busquier and S. Plaza, A construction of attracting periodic orbits for some classical third-order iterative methods, J. Comput. Appl. Math., 189, Nos 1-2 (2006), 22-33; https://doi.org/10.1016/j.cam.2005.03.049.

[4] K. Baranski, N. Fagella, X. Jarque and B. Karpinska, On the connectivity of the Julia sets of meromorphic functions, Invent. Math., 198, No 3 (2014), 591-636; https://doi.org/10.1007/s00222-014-0504-5.

[5] K. Baranski, N. Fagella, X. Jarque and B. Karpinska, Accesses to infinity from Fatou components, Trans. Amer. Math. Soc., 369, No 3 (2017), 18351867; https://doi.org/10.1090/tran/6739.

[6] K. Baranski, N. Fagella, X. Jarque and B. Karpinska, Connectivity of Julia sets of Newton maps: a unified approach, Rev. Mat. Iberoam., 34, No 3 (2018), 1211-1228; http://dx.doi.org/10.4171/rmi/1022.

[7] R.W. Barnard, J. Dwyer, E. Williams and G.B. Williams, Conjugacies of the Newton maps of rational functions, Complex Var. Elliptic Equ., 64, No 10 (2019), 16661685; https://doi.org/10.1080/17476933.2018.1547285.

[8] A.M. Benini and N. Fagella, Singular values and bounded Siegel disks, Math. Proc. Cambridge Philos. Soc., 165, No 2 (2018), 249-265; https://doi.org/10.1017/S0305004117000469.

[9] B. Bera and M.G.P. Prasad, Dynamics of two parameter family of hyperbolic sine like functions, J. Anal., 27, No 3 (2019), 895-912; https://doi.org/10.1007/s41478-018-0153-y.

[10] B. Bera and M.G.P. Prasad, Fixed points and dynamics of two-parameter family of hyperbolic cosine like functions, J. Math. Anal. Appl., 469, No 2 (2019), 1070-1079; https://doi.org/10.1016/j.jmaa.2018.09.052.

[11] W. Bergweiler, Lebesgue measure of Julia sets and escaping sets of certain entire functions, Fund. Math., 242, No 3 (2018), 281301; https://doi.org/10.4064/fm477-11-2017.

[12] H. Bruin, S. Luzzatto and S. van Strien, Decay of correlations in onedimensional dynamics, Ann. Scient. École Norm. Sup., 36, No 4 (2003), 621-646. 
[13] H. Bruin and D. Schleicher, Hausdorff dimension of biaccessible angles for quadratic polynomials, Fund. Math., 238 (2017), 201-239; http://dx.doi.org/10.4064/fm276-6-2016.

[14] J. Canela, Singular perturbations of Blaschke products and connectivity of Fatou components, Discrete Contin. Dyn. Syst., 37, No 7 (2017), 35673585; http://dx.doi.org/10.3934/dcds.2017153.

[15] J. Canela, N. Fagella and A. Garijo, Tongues in degree 4 Blaschke products, Nonlinearity, 29 (2016), 34643495; http://dx.doi.org/10.1088/0951$7715 / 29 / 11 / 3464$.

[16] T.K. Chakra, G. Chakraborty and T. Nayak, Herman rings with small periods and omitted values, Acta Math. Sci. Ser. B (Engl. Ed.), 38, No 6 (2018), 1951-1965; https://doi.org/10.1016/S0252-9602(18)30858-0.

[17] N. Chen, Y. Chen and K.W. Chung, Fractals from nonlinear IFSs of the complex mapping family $f(z)=z^{n}+c$, Fractals, 26, No 4 (2018), \# 1850044; https://doi.org/10.1142/S0218348X18500445.

[18] A. Chéritat and P. Roesch, Herman's condition and Siegel disks of bicritical polynomials, Commun. Math. Phys., 344, No 2 (2016), 397426; http://dx.doi.org/10.1007/s00220-016-2614-y.

[19] J.S. Christiansen, C. Henriksen, H.L. Pedersen and C.L. Petersen, Julia sets of orthogonal polynomials, Potential Anal., 50, No 3 (2019), 401-413; https://doi.org/10.1007/s11118-018-9687-5.

[20] J. T. Collins, Constructing attracting cycles for Halley and Schroder maps of polynomials, Discrete Contin. Dyn. Syst., 37, No 10 (2017), 5455-5465; https://doi.org/10.3934/dcds.2017237.

[21] A. Connes, E. Mcdonald, F. Sukochev and D. Zanin, Conformal trace theorem for Julia sets of quadratic polynomials, Ergod. Th. \& Dynam. Sys., 39, No 9 (2019), 2481-2506; https://doi.org/10.1017/etds.2017.124.

[22] R.L. Devaney, A Mandelpinski maze for rational maps of the form $z^{n}+\lambda / z^{d}$, Indagationes Mathematicae, 27 (2016), 1042-1058; http://dx.doi.org/10.1016/j.indag.2015.10.004.

[23] A. Douady and J.H. Hubbard, A proof of Thurston's topological characterization of rational functions, Acta Math., 171, No 2 (1993), 263-297; https://doi.org/10.1007/BF02392534. 
[24] A. Dudko and M. Yampolsky, Poly-time computability of the Feigenbaum Julia set, Ergod. Th. \& Dynam. Sys., 36, No 8 (2016), 2441-2462; http://dx.doi.org/10.1017/etds.2015.24.

[25] J. Fu and Y. Zhang, Connectivity of the Julia sets of singularly perturbed rational maps, Proc. Indian Acad. Sci. Math. Sci., 129, No 3 2019), Art. 32, 8 pp; https://doi.org/10.1007/s12044-019-0478-8.

[26] M. Garcia-Olivo, J.M. Gutiérrez and Á.A. Magreñán, A complex dynamical approach of Chebyshevs method, SeMA Journal, 71 (2015), 57-68; https://doi.org/10.1007/s40324-015-0046-9.

[27] A. Garijo and S. Godillon, On McMullen-like mappings, J. Fractal Geom., 2 (2015), 249-279; http://dx.doi.org/10.4171/JFG/21.

[28] L. Geyer, Linearizability of saturated polynomials, Indiana Univ. Mathematics J., 68, No 5 (2019), 15511578; https://doi.org/10.1512/iumj.2019.68.6160.

[29] S. Godillon, A family of rational maps with buried Julia components, Ergod. Th. \& Dynam. Sys., 35, No 6 (2015), 1846-1879; http://dx.doi.org/10.1017/etds.2014.22.

[30] J. Graczyk and S. Smirnov, Non-uniform hyperbolicity in complex dynamics, Invent. Math., 175, No 2 (2009), 335-415; https://doi.org/10.1007/s00222-008-0152-8.

[31] J. Hawkins and M.M. Rocha, Dynamics and Julia set of iterated elliptic functions, New York J. Math., 24 (2018), 947979.

[32] S. Hittmeyer, B. Krauskopf and H.M. Osinga, Interactions of the Julia set with critical and (un)stable sets in an angle-doubling map on $\mathbb{C} \backslash\{0\}$, Internat. J. Bifur. Chaos, 25, No 4 (2015), \# 1530013, 36 pp; https://doi.org/10.1142/S021812741530013X.

[33] G. Honorato, Dynamics and limiting behavior of Julia sets of König's method for multiple roots, Topology Appl., 233 (2018), 16-32; https://doi.org/10.1016/j.topol.2017.10.013.

[34] K. Katagata, Entire functions whose Julia sets include any finitely many copies of quadratic Julia sets, Nonlinearity, 30, No 6 (2017), 23602380; https://doi.org/10.1088/1361-6544/aa6c01. 
[35] K. Katagata, Transcendental entire functions whose Julia sets contain any infinite collection of quasiconformal copies of quadratic Julia sets, Discrete Contin. Dyn. Syst., 39, No 9 (2019), 53195337; http://dx.doi.org/10.3934/dcds.2019217.

[36] M. Kisaka, Topological properties of Fatou sets and Julia sets of transcendental entire functions, Sgaku, 65, No 3 (2013), 269298; https://doi.org/10.11429/sugaku.0653269.

[37] M. Kisaka, On topological properties of Fatou sets and Julia sets of transcendental entire functions, Sugaku Expositions, 30, No 2 (2017), 235273; https://doi.org/10.1090/suga/425.

[38] Y.H. Liang, W.R. Wu and J. Juang, Fastest synchronized network and synchrony on the Julia set of complex-valued coupled map lattices, Discrete Contin. Dyn. Syst. Ser. B, 21, No 1 (2016), 173-184; http://dx.doi.org/10.3934/dcdsb.2016.21.173.

[39] K.A. Lindsey, Shapes of polynomial Julia sets, Ergod. Th. \& Dynam. Sys., 35, No 6 (2015), 1913-1924; http://dx.doi.org/10.1017/etds.2014.8.

[40] L. Lomonaco, Parabolic-like mappings, Ergod. Th. \& Dynam. Sys., 35, No 7 (2015), 2171-2197; http://dx.doi.org/10.1017/etds.2014.27.

[41] L. Ma, A continuity result on quadratic matings with respect to parameters of odd denominator rationals, Math. Proc. Cambridge Philos. Soc., 167, No 2 (2019), 369-388; https://doi.org/10.1017/S0305004118000397.

[42] C.T. McMullen, Automorphisms of rational maps. In: Holomorphic Functions and Moduli, D. Drasin and I. Kra and C.J. Earle and A. Marden and F.W. Gehring (Eds.), Vol. 1, pp. 31-60. Mathematical Sciences Research Institute Publications, Vol. 10, Springer, New York (1988); https://doi.org/10.1007/978-1-4613-9602-4_3.

[43] D. Meyer, Expanding Thurston maps as quotients, arXiv:0910.2003v2 (2012); https://arxiv.org/abs/0910.2003v2.

[44] D. Meyer, Invariant Peano curves of expanding Thurston maps, Acta Math., 210 (2013), 95-171; https://doi.org/10.1007/s11511-013-0091-0.

[45] D. Meyer, Unmating of rational maps: Sufficient criteria and examples. In: Frontiers in Complex Dynamics, A. Bonifant (Ed.), 198-233; In celebration of John Milnors 80th birthday. Based on a conference, Banff, 
Canada, February 2011. Princeton, NJ, Princeton University Press (2014); https://doi.org/10.1515/9781400851317-012.

[46] J. Milnor, Dynamics in One Complex Variable, Friedrick Vieweg and Son (2000).

[47] L.K. Mork, T. Vogt, K. Sullivan, D. Rutherford and D.J. Ulness, Exploration of filled-in Julia sets arising from centered polygonal lacunary functions, Fractal Fract., 3, No 3 (2019), 42 pp.; http://dx.doi.org/10.3390/fractalfract3030042.

[48] S. Morosawa, Semigroups whose Julia sets are Cantor targets, Complex Var. Elliptic Equ., 64, No 4 (2019), 701709; https://doi.org/10.1080/17476933.2018.1481834.

[49] S. Morosawa, Y. Nishimura, M. Taniguchi and T. Ueda, Holomorphic Dynamics, Cambridge University Press, Cambridge (2000).

[50] D.A. Nicks and D.J. Sixsmith, The bungee set in quasiregular dynamics, Bull. Lond. Math. Soc., 51, No 1 (2019), 120-128; http://dx.doi.org/10.1112/blms.12215.

[51] J.W. Osborne, P.J. Rippon and G.M. Stallard, The iterated minimum modulus and conjectures of Baker and Eremenko, J. Anal. Math., 139, No 2 (2019), 521558; https://doi.org/10.1007/s11854-019-0065-z.

[52] L. Qiu, Z.X. Xuan and Y. Zhao, Radial distribution of Julia sets of some entire functions with infinite lower order, Chinese Ann. Math. Ser. A, 40, No 3 (2019), 325334; http://dx.doi.org/10.16205/j.cnki.cama.2019.0024.

[53] W. Qiu, F. Yang and Y. Yin, Quasisymmetric geometry of the Cantor circles as the Julia sets of rational maps, Discrete Contin. Dyn. Syst. Ser. A, 36, No 6 (2016), 3375-3416; http://dx.doi.org/10.3934/dcds.2016.36.3375.

[54] W. Qiu, F. Yang and Y. Yin, Quasisymmetric geometry of the Julia sets of McMullen maps, Sci. China Math., 61, No 12 (2018), 22832298; https://doi.org/10.1007/s11425-016-9228-x.

[55] P.J. Rippon and G.M. Stallard, Transcendental Dynamics and Complex Analysis, London Mathematical Society Lecture Note Series, 348, Cambridge University Press, Cambridge (2008). 
[56] J. Rivera-Letelier and W. Shen, Statistical properties of one-dimensional maps under weak hyperbolicity assumptions, Ann. Scient. École Norm. Sup., 47, No 6 (2014), 1027-1083.

[57] M. Sajid, Singular values and real fixed points of one-parameter families associated with fundamental trigonometric functions $\sin z, \cos z$ and $\tan z$, Int. J. Appl. Math., 33, No 4 (2020), 635-647; http://dx.doi.org/10.12732/ijam.v33i4.8.

[58] M. Sajid and G.P. Kapoor, Chaos in dynamics of a family of transcendental meromorphic functions, J. of Nonlinear Analysis and Application, 2017, No 1 (2017), Art. ID jnaa-00355, 11 pp.; http://dx.doi.org/10.5899/2017/jnaa-00355.

[59] T. Sharland, Matings of cubic polynomials with a fixed critical point, Part I: Thurston obstructions, Conform. Geom. Dyn., 23 (2019), 205220; https://doi.org/10.1090/ecgd/342.

[60] B.H. Subedi and A. Singh, Fatou, Julia, and escaping sets in holomorphic (sub)semigroup dynamics, Turkish J. Math., 43, No 2 (2019), 930940; http://dx.doi.org/10.3906/mat-1810-133.

[61] F. Yang, A criterion to generate carpet Julia sets, Proc. Amer. Math. Soc., 146, No 5 (2018), 21292141; https://doi.org/10.1090/proc/13924.

[62] J. Yang, Mating the basilica with a Siegel disk, Conform. Geom. Dyn., 19 (2015), 258297; https://doi.org/10.1090/ecgd/284.

[63] S. Zakeri, Rotation Sets and Complex Dynamics, Springer (2018); https://doi.org/10.1007/978-3-319-78810-4.

[64] G. Zhang, On PZ type Siegel disks of the sine family, Ergod. Th. \& Dynam. Sys., 36, No 3 (2016), 973-1006; http://dx.doi.org/10.1017/etds.2014.89. 
